\title{
Correction to: Thoracotomy as the Surgical Route for Synchronous Thoracic and Non-thoracic Procedures
}

\author{
Achilleas G. Lioulias, Michail D. Tsimpinos \\ and Meletios A. Kanakis
}

\section{Correction to: \\ Chapter "Thoracotomy as the Surgical Route for Synchronous Thoracic and Non-thoracic Procedures" in: C. E. Nistor et al. (eds.), Thoracic Surgery, https://doi.org/10.1007/978-3-030-40679-0_73}

In the original version of the book, the spelling of the author name Meletios A. Kanakis and his affiliation was incorrect.

The corrected name and affiliation is given below:

Meletios A. Kanakis, Department of Paediatric and Congenital Heart Surgery, Onassis Cardiac Surgery Center, Athens, Greece

The correction chapter and the book have been updated. 